\title{
Problems derived from the simultaneous utilisation of different flickermeters in large electric networks
}

\author{
Eguíluz, L.I.; Mañana, M; Ortiz, A.; Madrazo, A. \\ Department of Electrical Engineering \\ E.T.S.I.I., University of Cantabria \\ Avda. Los Castros s/n, 39006 Santander (Spain) \\ phone:+34 942201378, fax:+34 942201385, e-mail: eguiluzi@unican.es
}

\begin{abstract}
Power Quality assessment in distribution networks requires distributed measurements in order to verify the PQ state of the art. This process cannot simply be considered as an extension of a measurement at a given point because several variables need to be considered: number of PQM; election of optimal measurement location; synchronisation of instrumentation and management of captured data.

This work focuses on the problems related with the simultaneous utilisation of different flickermeters in large electric networks.

From a classical instrumentation point of view, several flickermeters from different customers are tested in the laboratory in order to determine the differences in the accuracy of the results. Both Pst and Plt are statistical parameters calculated using a large number of samples of the instantaneous flicker level $\mathrm{I}_{\mathrm{FL}}$. The number of captured samples can vary from one instrument to another, so different flickermeters provide different values.
\end{abstract}

\section{Key words}

Power Quality, flicker, instrumentation, measuring large distribution networks.

\section{Introduction}

Flicker measurement is not an easy task when it is undertaken in large distribution networks. The standard EN 50160 [1] establishes the maximum value that the 95 th percentile must have in order to comply with the legislation.

However, there are no documents explaining how to choose the optimal location for the instrumentation. Another additional problem the users have to deal with is the use of different flickermeters built by different manufacturers. This research work focuses on this problem, analysing it and providing some solutions.

\section{Power Quality instrumentation}

The set of Power Quality Instrumentation devices under test (PQDUT) includes five flickermeters from four different companies. Four recorders are designed according to the standard EN 61000-4-15 [2] using a digital implementation. The last one is based on IEC 868 [3] and is implemented using analog filters.

Table I summarises some functional and design characteristics of the PQDUT.

TABLE I. - PQDUT functional and design characteristics.

\begin{tabular}{|l|l|}
\hline Flickermeter & Description \\
\hline M_1 & $\begin{array}{l}\text { Three phases. } \\
\text { Digital. } \\
\text { EN 50160. }\end{array}$ \\
\hline M_2 & $\begin{array}{l}\text { Three phases. } \\
\text { Digital. } \\
\text { EN 61000-4-15. }\end{array}$ \\
\hline M_3 & $\begin{array}{l}\text { One phase. } \\
\text { Digital. } \\
\text { EN 61000-4-15. }\end{array}$ \\
\hline M_4 & Three phases. \\
& Digital \\
\hline M_5 & Three phases. \\
& Analog. \\
IEC 868.
\end{tabular}

\section{Test system and methodology}

Tests has been performed following two different procedures:

1. All the flickermeters under test (FUD) were connected together to the same point of the low voltage distribution network.

2. All the FUD were connected together to an arbitrary waveform power supply and subjected to test HP 6834B [4] which can be programmed in order to generate a $50 / 60 \mathrm{~Hz}$ sinuoidal voltage with or without distortion. The ac source combines three instruments in one unit as shown in Figure 1. The function generator produces waveforms with user-defined amplitude, frequency and shape. The power amplifier amplifies the function generator signal to 
produce the ac voltage. No power generation capabilities are needed because the flickermeters do not have any power consumption. The measurement functions range from a simple readback of rms voltage and current, to sophisticated capabilities such as waveform analysis.

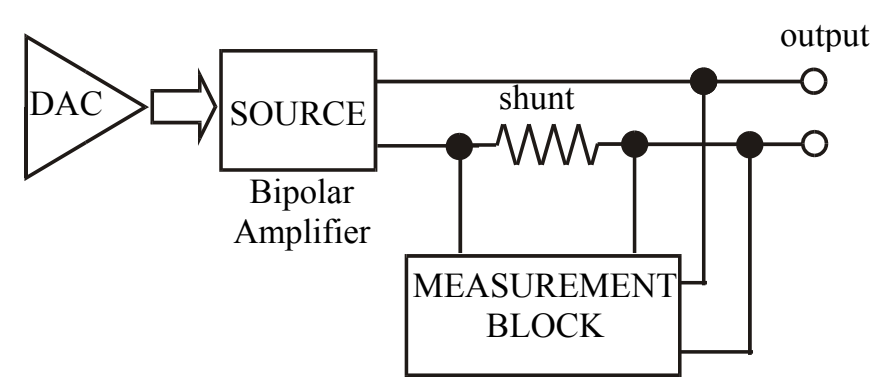

Powermeter

FFT Analyzer

Fig. 1. AC Source Functional Elements.

All the evaluated instrumentation has been designed according to IEC 868 [3] or the new standard revision EN 61000-4-15 [2] so none of them can be considered as a calibration reference. In order to obtain accuracy in the analysis of the results, a statistical approach has been used so that FUD are compared with mean values. Only one of the five instruments has been designed using analog electronics. The rest are completely digital so the need for calibration is reduced to the voltage adapter subsystem located before the analog to digital converter. Digital circuits provide the implementation with more stability.

\section{Methodology}

In order to compare the results obtained for the five different flickermeters, a statistical approach has been followed.

If we consider PST(t) as a time dependent random variable, the average value at instant $t$ is

$\underset{\operatorname{PST}}{-}(t)=\frac{\sum_{i=1}^{N} P S t_{i}(t)}{N}$

where,

Pst $_{i}(t) \quad$ Pst of flickermeter $i$ at interval t. All the devices have the same weight.

N Number of evaluated flickermeters.

The average is a simple and popular estimate of location. If the data sample comes from a normal distribution, then the sample average is also optimal [5]. The problem appears when real data contains data entry errors or glitches because some bad data values can move the average away from the centre of the rest of the data. The average computed as the 50 th percentile of the sample can help to reduce the problem. The idea behind the median is to ignore a small percentage of the highest and lowest values when we are computing the centre of the sample. This procedure is useful when then number of samples is high enough. Unfortunately the experiment has been carried out with five flickermeters so the data population is quite small.

The dispersion is measured using the variance and the standard deviation which are optimal for normally distributed samples.

The range, computed as the difference between the maximum and minimum values, can also be used but it is not robust to outliers. The variance of PST( $t)$ is

$\sigma_{P s t}^{2}(i)=\frac{\sum_{i=1}^{N}\left(P s t_{i}(t)-P \overline{S T}(i)\right)^{2}}{N}$

The standard deviation is the square root of the variance $\sigma_{P s t}^{2}(i)$ and is also a function of time. It has the desirable property of being in the same units as the data.

Equations (1) and (2) are time dependent, so that all the flickermeters must be synchronised. The need for exact synchronisation can be overcome using other analysis techniques such as the probability density function (pdf) or the cumulative distribution function (cdf). However, these functions lose time dependency so error evaluation has only global meaning.

The Pst discrete probability density function (pdf) gives the probability of observing a particular value of the Pst during a time interval.

If pdf is the discrete probability density function for the random variable Pst, the associated cumulative distribution function cdf can be obtained using the following expression:

$$
c d f(\operatorname{PST}(t))=P(P s t \leq x)=\sum_{j=1}^{J_{x}} p d f(j)
$$

where,

$$
\mathrm{J}_{\mathrm{x}} \quad \text { number of intervals. }
$$

It is well known that cdf has two theoretical properties:

1. The cdf ranges from 0 to 1 .

2. If $y>x$, then the cdf of $y$ is greater than or equal to the cdf of $x$.

\section{Results}

Firstly, some laboratory tests have been performed in order to compare the values provided by the flickermeters. 
In the first test, the flicker level has been measured without any input signal. Figure 2 shows the Pst time evolution. Only two instruments noticed that no voltage was applied to their inputs. What is more, one of them provides a value of Pst equal to 0.15 .

In the second test, a $50 \mathrm{~Hz}$ sinusoidal voltage supply by the programmable power generator has been applied in order to know the steady state value provided by the flickermeters when no flicker exists. Figure 3 shows the time evolution of Pst for the all flickermeters.

Pst

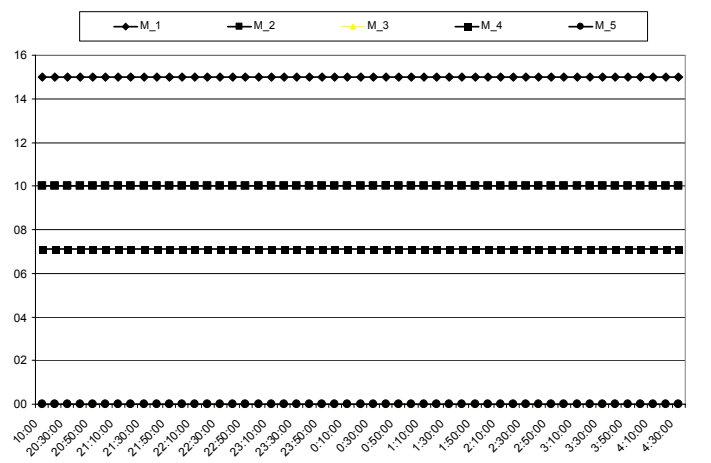

Time

Fig. 2. Pst evolution without any input signal. Pst

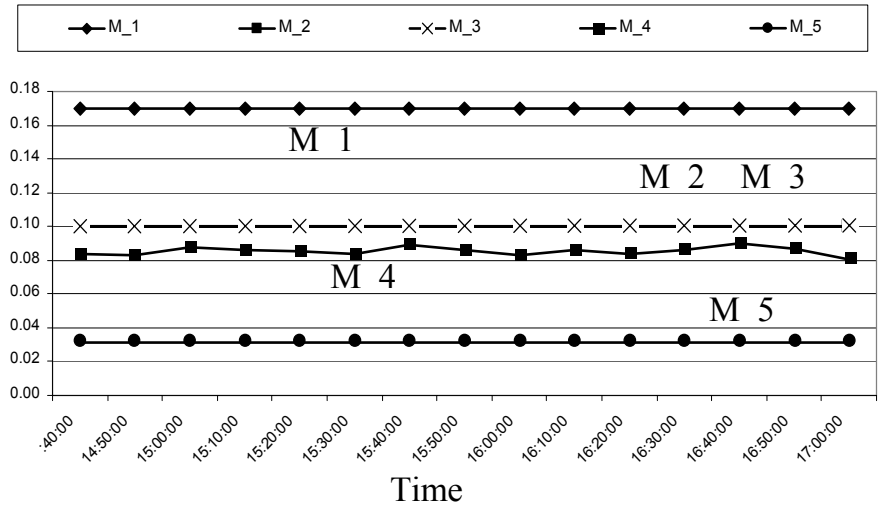

Fig. 3. Pst evolution with a $230 \mathrm{~V}, 50 \mathrm{~Hz}$ input signal.

The average and standard deviation are summarised in Table II.

Table II. Pst statistical data registered by the flickermeters with a sinusoidal input signal.

\begin{tabular}{|l|l|l|l|}
\hline Meter & Average & $\sigma^{2}$ & $\sigma$ \\
\hline M_1 & 0.17 & 0.0 & 0.0 \\
\hline M_2 & 0.1 & 0.0 & 0.0 \\
\hline M_3 & 0.1 & 0.0 & 0.0 \\
\hline M_4 & 0.085 & 0.0 & 0.0 \\
\hline M_5 & 0.03 & 0.0 & 0.0 \\
\hline
\end{tabular}

In order to obtain the differences between the different flickermeters, all the devices were connected to the same point in the low voltage distribution network. Figure 4 shows the Pst values captured by the instrumentation.
The distribution $P S T(t)$ has been computed using equation (1). Once all the $P s t_{i}(t)$ were calculated, they were compared with it in order to evaluate the error at each instant $t$. The result is shown in Figure 5.

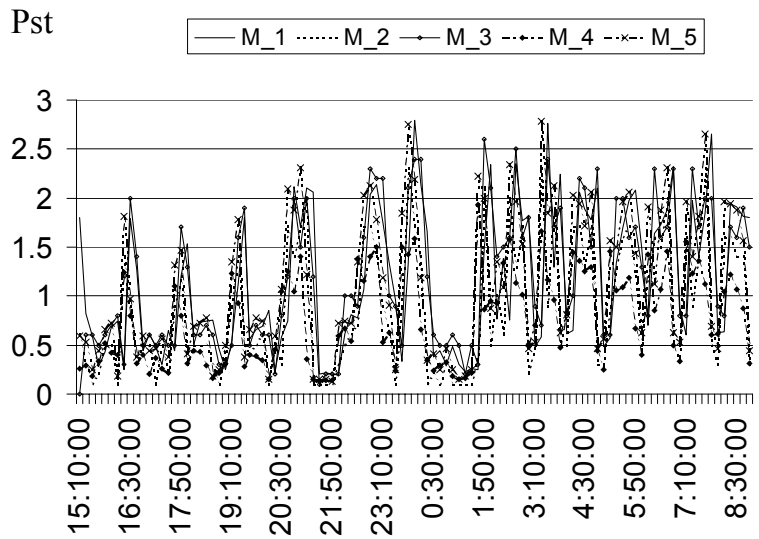

Time (hour)

Fig. 4. Pst evolution measured by different flickermeters.

Pst deviation

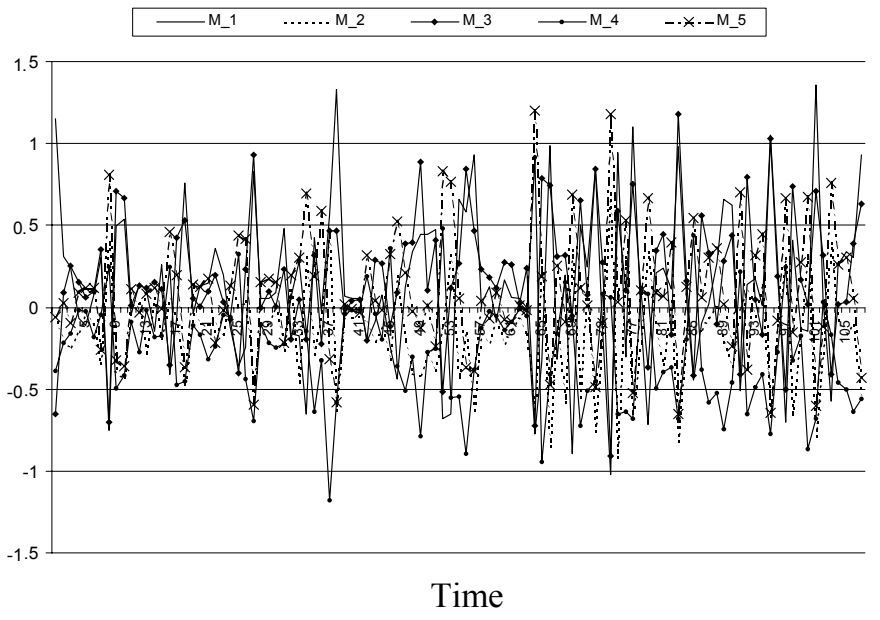

Fig. 5. Deviation of Pst from average distribution.

The average and standard deviation of each flickermeter with the average distribution is summarised in Table III.

Table III. Statistical values for the deviation of Pst from the computed average distribution.

\begin{tabular}{|l|l|l|l|}
\hline Meter & Average & $\sigma^{2}$ & $\sigma$ \\
\hline M_1 & 0.1313 & 0.2321 & 0.4818 \\
\hline M_2 & -0.1567 & 0.0907 & 0.3013 \\
\hline M_3 & 0.1816 & 0.1498 & 0.3871 \\
\hline M_4 & -0.2568 & 0.1158 & 0.3403 \\
\hline M_5 & 0.1006 & 0.1383 & 0.3720 \\
\hline
\end{tabular}

Although some previous work [6, 7] focused on the problem of simultaneous disturbance capturing, it is now clear that flicker measurements in the presence of sags and swells requires further analysis. This is because flicker algorithms according to IEC 868 and EN 61000-415 are sensitive to rms variations. This problem is 
specially important when flicker is measured in dynamic loads such as arc furnaces. If the short circuit power at the common coupling point is not high enough in relation to the power of the arc furnace, the voltage close to the load will undergo the development of small sags and an increase in flicker level. This correlation can be easily assumed because flicker evaluation considers all spectral components ranging approximately from 0.5 to $35 \mathrm{~Hz}$. What should be considered carefully is the presence of one single sag because the 10 minute Pst will change. If it isn't clear the influence on humans of that single sag if we think in terms of light oscillation.

Figure 6 shows voltage rms value for phase a and Figures 7 and 8 show the voltage snapshot during the transient. In addition, Figures 9 and 10 show Pst and Plt evolution for phase a in a $12 \mathrm{kV}$ distribution network inside an arc furnace factory. It can be observed that there is a close correlation between rms values and Pst / Plt.

Rms Va (V)

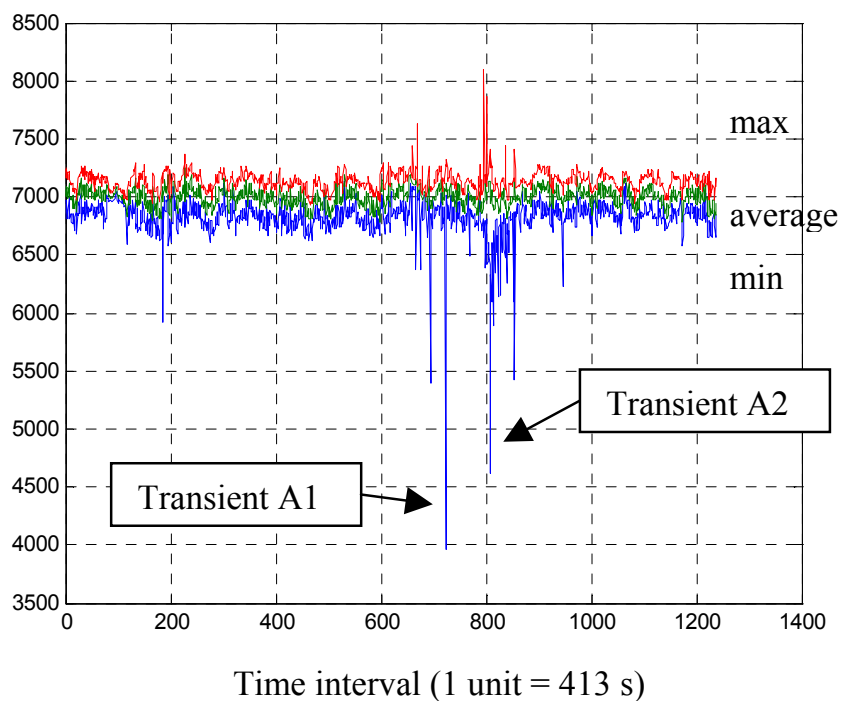

$\mathrm{Va}(\mathrm{V})$

Fig. 6. rms evolution in phase a.

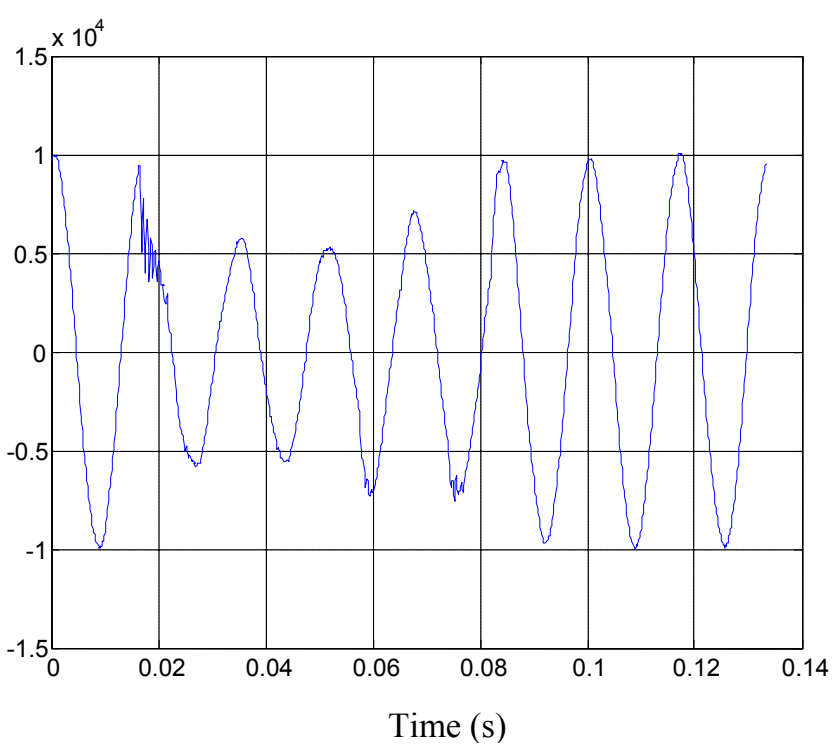

Fig. 7. Voltage transient A1 at phase a.
$\mathrm{Va}(\mathrm{V})$

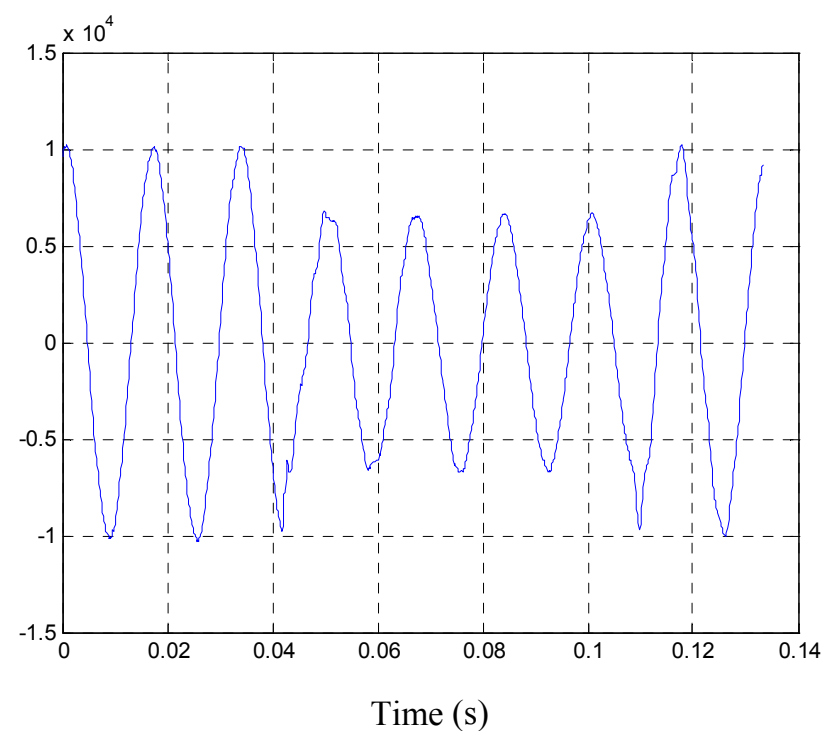

Pst Fig. 8. Voltage transient A2 at phase a.

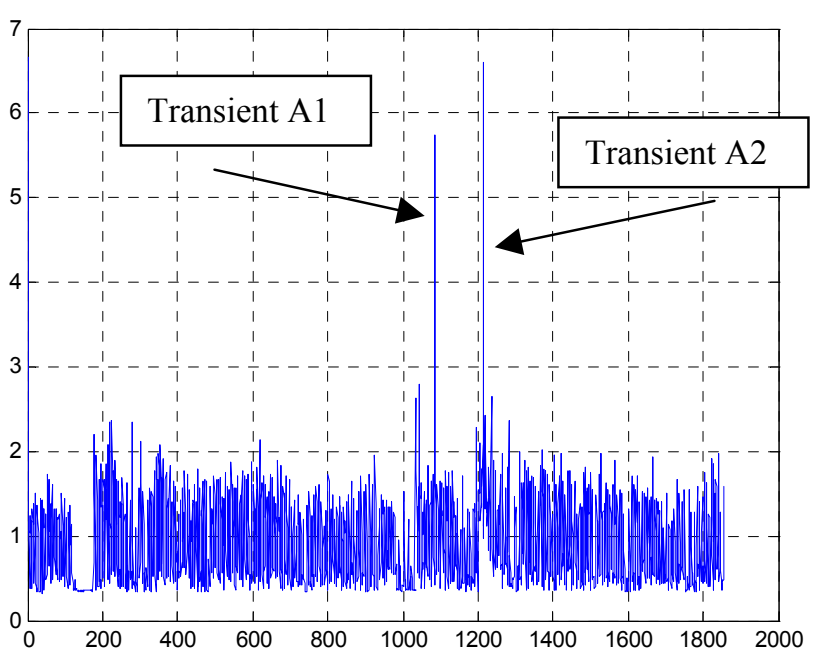

Time interval $(1$ unit $=10$ minutes $)$

Fig. 9. Pst evolution in phase a.

Plt

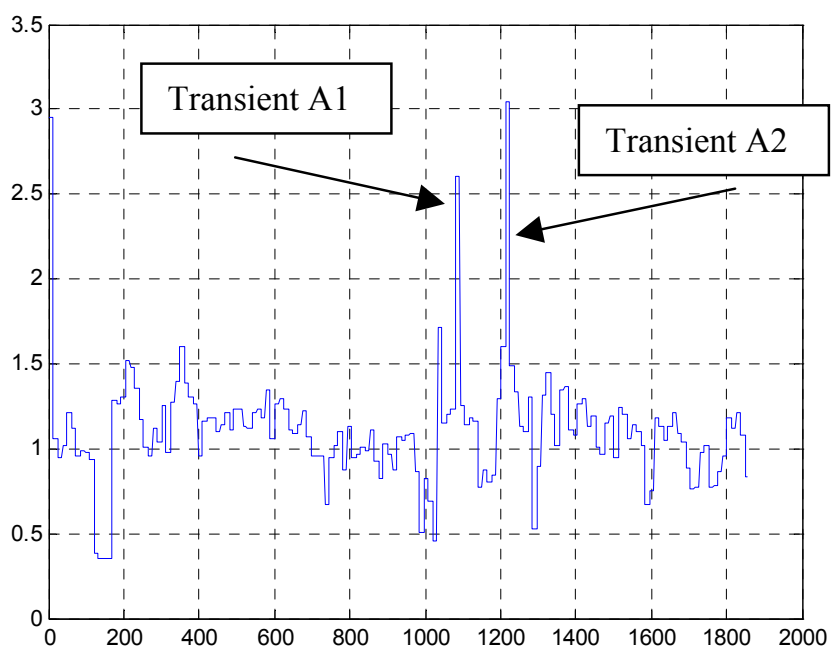

Time interval ( 1 unit $=10$ minutes $)$

Fig. 10. Plt evolution in phase a. 
IEC is developing a new standard document providing support on power quality measurement. The draft contains procedures to follow when simultaneous disturbances appear [8].

\section{Conclusions}

If different flickermeters are used it is necessary to test simultaneously all the devices in order to determine steady state maximum deviations.

The location of flickermeters in low voltage distribution networks should avoid sharing the same distribution transformer. In fact it is worth considering different substations in order to verify how flicker propagates over the distribution network.

The synchronisation of the instrumentation is not a trivial task because instrumentation manufactures do not provide technical information about the initialisation process. Some equipment requires an initialisation period after the starting of the measurement process. However, from a statistical point of view, delays between data samples are not important so EN 5016095 th percentiles can be computed with different devices without any special considerations.

\section{References}

[1] CENELEC EN 50160.Voltage characteristics of electricity supplied by public distribution systems. 1999.

[2] CENELEC EN 61000-4-15. Electromagnetic compatibility (EMC) -- Part 4-15: Testing and measurement techniques - Flickermeter - Functional and design specifications. 1998.

[3] IEC 868. IEC/TR2 60868 (1986-09). Flickermeter Functional and design specifications.

[4] HP6834B. User's Guide. Agilent Technologies. 2000.

[5] Using Matlab 6.0 - Simulink 5.0. Mathworks. 2002.

[6] Koponen, P.; Voltage dips cause problems with digital flickermeters. IEEE Power Tech Conference. Vol. 1. September 10-13, Porto, Portugal. 2001.

[7] Mañana, M.; Eguíluz, L.I.; Lavandero, J.C.; Díez, G.; Influence on Power Quality Measurement of the Simultaneous Presence of Different Disturbances. Approved for their presentation in ICREPQ'2003 (International Conference on Renewable Energy and Power Quality). 9-11 April 2003. Vigo, Spain.

[8] IEC 61000-4-30 77A/333/CD. Electromagnetic Compatibility. Part 4. Section 30: Testing and measurement techniques. Power Quality measurement methods. (draft). 